Wolf-Rayet Phenomena in Massive Stars and Starburst Galaxies

Proceedings IAU Symposium No. 193, (c) 1999 IAU

K.A. van der Hucht, G. Koenigsberger \& P.R.J. Eenens, eds.

\title{
The stellar content of NGC 2363 and its surroundings
}

Laurent Drissen, Jean-René Roy, Carmelle Robert, and Daniel Devost

Département de Physique and Observatoire du Mont Mégantic, Université Laval, Québec, Qc G1K YP4, Canada

Abstract. A detailed study of the stellar content of the giant $\mathrm{H}$ II region NGC 2363 and its surroundings in the Magellanic galaxy NGC 2366 is undertaken to understand the starburst phenomenon.

\section{Discussion}

The highest surface brightness $\mathrm{H}$ II region in the sky, NGC 2363, is located at the southern end of the bar in the nearby Magellanic galaxy NGC 2366. Its unusual combination of kinematical features (Roy et al. 1992) make it the archetype of a whole class of small but powerful young starbursts with blowout-like properties. In order to understand the star formation process in starbursts, we have gathered an extensive dataset of images with HST-WFPC2 and CFHT-MONICA, as well as UV spectra (HST-FOS and -STIS) of NGC 2363 and its surroundings.

Two star clusters are responsible for the ionization of NGC 2363 (Figure 1). Wolf-Rayet stars are known to be present in cluster B (Drissen et al. 1993) Indeed, our narrow-band (F469N) images reveal the presence of three individual

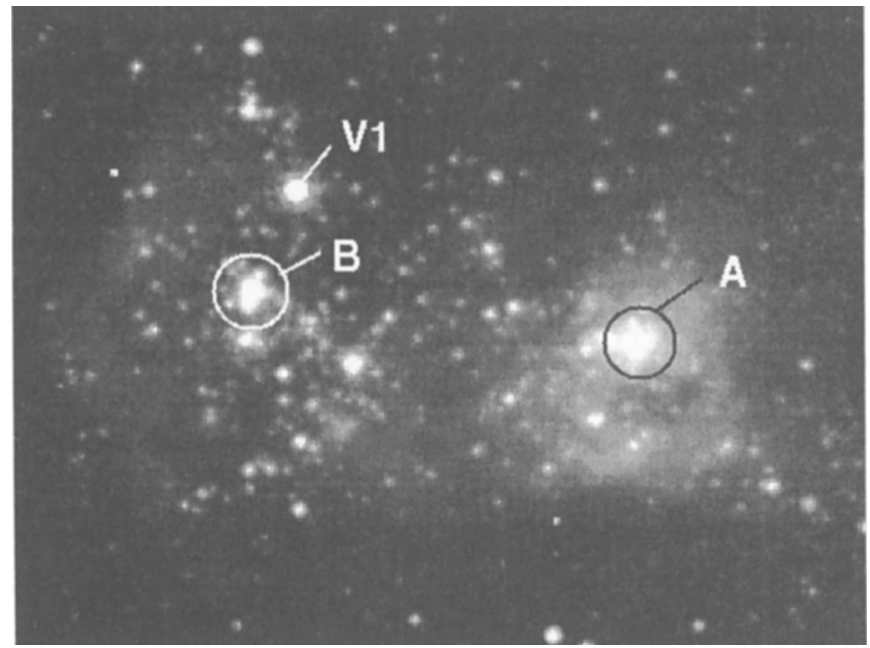

Figure 1. HST-WFPC2 $V$ image of NGC 2363, showing the clusters A and $\mathrm{B}$, as well as the erupting LBV V1. The field is $10^{\prime \prime} \times 8^{\prime \prime}(170 \times 140 \mathrm{pc})$. North is up, East to the left. 


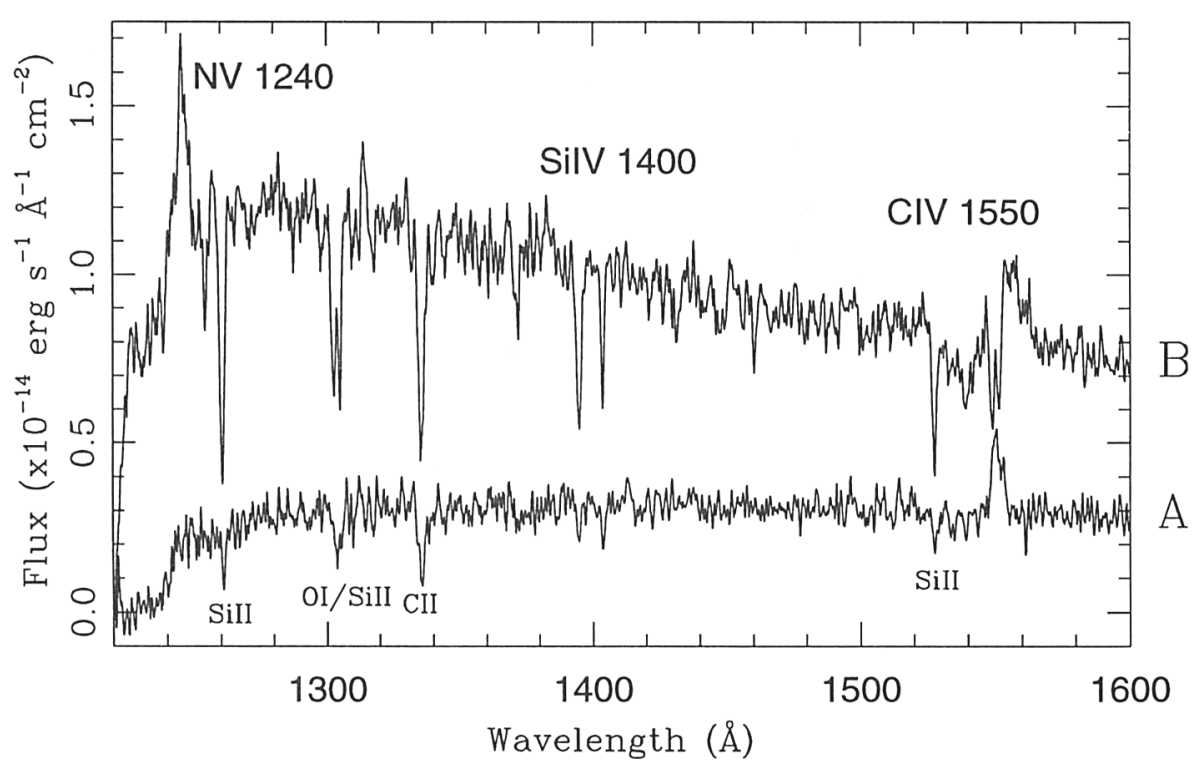

Figure 2. HST-FOs spectrograms of clusters A and B in NGC 2363. The spectrum of cluster $\mathrm{A}$ is flat and contains only interstellar narrow absorption features and the nebular C IV $\lambda 1550$ emission line. Prominent P-Cygni profiles of $\mathrm{N} \mathrm{V} \lambda 1240$ and C IV $\lambda 1550$ are seen in the spectrum of cluster B, typical of massive stars with strong winds.

WR stars within $0 ! 3$ of its core. The presence of the WR stars, the erupting LBV V1 (Drissen et al. 1997) and two massive O-type stars (whose STIS spectra were obtained at the same time as those of V1), the shape of the UV lines in the FOS spectrum (Figure 2) and the expansion velocity of the surrounding bubble, suggest that cluster $\mathrm{B}$ is $\sim 4$ to $6 \mathrm{Myr}$ old. The absence of stellar wind features in the spectrum of knot $\mathrm{A}$, the extreme nebular surface brightness close to its core, and its IRAS colors show that knot A is a very young $(<1 \mathrm{Myr})$ and dense cluster of massive stars still embedded in their natal molecular clouds. The giant $\mathrm{H}$ il region located $300 \mathrm{pc}$ to the east of NGC 2363 (NGC 2366-II) is much older than NGC 2363: no WR stars are found, but a dozen red supergiants are detected, suggesting an age of $\sim 10 \mathrm{Myr}$.

It is tempting to suggest that the extended group of stars to the southwest of NGC 2366 is a satellite galaxy and that its passage through the bar has triggered the wave of star formation portrayed by NGC 2366-II and NGC 2363.

\section{References}

Drissen, L., Roy, J.-R., Moffat, A.F.J. 1993, AJ 106, 1460

Drissen, L., Roy, J.-R., Robert, C. 1997, ApJ 474, L35

Roy, J.-R., Aubé, M., McCall, M.L., Dufour, R.J. 1992, ApJ 386, 498 